\title{
Seiberg-Witten transforms of noncommutative solitons
}

\author{
Koji Hashimoto* \\ Institute for Theoretical Physics, University of California, Santa Barbara, California 93106-4030 \\ Hirosi Ooguri ${ }^{\dagger}$ \\ Institute for Theoretical Physics, University of California, Santa Barbara, California 93106-4030 \\ and California Institute of Technology 452-48, Pasadena, California 91125
}

(Received 12 June 2001; published 3 October 2001)

\begin{abstract}
We evaluate the Seiberg-Witten map for solitons and instantons in noncommutative gauge theories in various dimensions. We show that solitons constructed using the projection operators have delta-function supports when expressed in the commutative variables. This gives a precise identification of the moduli of these solutions as locations of branes. On the other hand, an instanton solution in four dimensions allows deformation away from the projection operator construction. We evaluate the Seiberg-Witten transform of the $U(2)$ instanton and show that it has a finite size determined by the noncommutative scale and by the deformation parameter $\rho$. For large $\rho$, the profile of the D0-brane density of the instanton agrees surprisingly well with that of the Belavin-Polyakov-Schwarz-Tyupkin (BPST) instanton on commutative space.

DOI: 10.1103/PhysRevD.64.106005 PACS number(s): 02.40.Gh, 05.45.Yv, 11.15.-q, 11.25.-w
\end{abstract}

\section{INTRODUCTION}

Noncommutative gauge theories can be realized by considering branes in string theory with a constant NeveuSchwarz-Neveu-Schwarz two-form field [1]. It is described by noncommutative gauge fields $\hat{A}_{i}$ on a noncommutative space whose coordinates obey the commutation relation

$$
\left[\hat{x}^{i}, \hat{x}^{j}\right]=i \theta^{i j}
$$

One of the remarkable features of these theories is that there is a universal way to construct a large class of classical solutions [2-45]. In particular, in two dimensions, all solutions to the noncommutative Yang-Mills equations with gauge group $U(N)$ are classified in Ref. [15], and it was shown that they take the form

$$
X^{i}=U \hat{x}^{i} U^{\dagger}+\sum_{a=1}^{m} \lambda_{a}^{i}|a\rangle\langle a| \quad(i=1,2),
$$

where

$$
X^{i}=\hat{x}^{i}-\theta^{i j} \hat{A}_{j}(\hat{x})
$$

are operators acting on the Hilbert space $\mathcal{H}$, which is the Fock space of Eq. (1.1) times $\mathbf{C}^{N},\{|a\rangle\}_{a=1 \ldots m}$ is an $m$-dimensional subspace of $\mathcal{H}$, and $U$ is the associated shift operator obeying

$$
U^{\dagger} U=1, \quad U U^{\dagger}=1-\sum_{a=1}^{m}|a\rangle\langle a| .
$$

\footnotetext{
*Email address: koji@itp.ucsb.edu

${ }^{\dagger}$ Email address: ooguri@theory.caltech.edu
}

Thus the solutions are parametrized by the rank $m$ of the projection operator $1-U U^{\dagger}$, the rank $N$ of the gauge group, ${ }^{1}$ and the $2 m$ moduli parameters $\lambda_{a}^{i}$. These solitons are interpreted as D0 branes on D2 branes with $m$ and $N$ being the D0 and D2 charges, respectively. There has been evidence suggesting that $\lambda_{a}^{i}$ correspond to the locations of the D0 branes $[11,15,50]$. In this paper we will confirm this interpretation using the Seiberg-Witten map. In higher dimensions, a complete classification of solutions has not been carried out, although some special solutions are known, such as instanton solutions in four dimensions, which can be interpreted as D0 branes on D4 branes $[2-5,12,13,44,16]$. These higherdimensional solutions do not necessarily take the form (1.2).

In Ref. [51], it was shown that there are two equivalent descriptions of the theory; one in terms of ordinary gauge fields $A_{i}$ on a commutative space, and another in terms of noncommutative gauge fields $\hat{A}_{i}$ on a noncommutative space. The map between $A_{i}$ and $\hat{A}_{i}$ is called the SeibergWitten map. In Refs. [46-48], an explicit expression for the Seiberg-Witten map was found for the $U(1)$ part of the field strength, by studying the coupling of the gauge field to the Ramond-Ramond potentials of closed string in the bulk. ${ }^{2}$ The

\footnotetext{
${ }^{1}$ It may not be evident in the expression (1.2) that the rank $N$ of gauge group is a parameter of the solution invariant under the $U(\infty)$ gauge symmetry. To see that there is a gauge invariant definition of $N$, we point out the formula derived in [46-48]:

$$
\operatorname{Tr}\left[\operatorname{Pf}\left(\left[X^{i}, X^{j}\right]\right) e^{i k \cdot X}\right]=N \delta(k) .
$$

This holds as far as the gauge field $\hat{A}_{i}(x)$ has a compact support when it is expressed in terms of commutative variables via the Seiberg-Witten map. One may also be able to show that $N$ is gauge invariant by using the more precise definition of the $U(\infty)$ group recently given in Ref. [49].

${ }^{2}$ There has also been an approach [52-55] to express the SeibergWitten map using the Kontsevich formal map [56,57].
} 
expression was conjectured earlier in Ref. [58]. It was proven in Ref. [46] that it indeed satisfies the conditions for the Seiberg-Witten map without relying on the string theory origin of the expression. In this paper, we evaluate the Seiberg-Witten map for the noncommutative soliton solutions in the above paragraph and express them in terms of the commutative variables.

In two dimensions, where a solution always takes the form (1.2), we find that the $U(1)$ part of the commutative field strength has a delta-function support at $x^{i}=\lambda_{a}^{i}$. This confirms the earlier observation that the moduli $\lambda_{a}^{i}$ should be regarded as positions of D0 branes on the D2 branes. It is interesting to note that $\lambda_{a}^{i}$ are commutative parameters even though they are describing the locations of the noncommutative solitons. A natural explanation for this is that the coordinates $x^{i}$ of the commutative variables $A_{i}(x)$ should be considered as the closed string coordinates, which are commutative, since the Seiberg-Witten map we use was derived from the study of the coupling of the gauge theory to the Ramond-Ramond potentials in the bulk. It is rather surprising that, whether the gauge group is Abelian or non-Abelian, all the solutions in two dimensions are singular when expressed in terms of the commutative variables $A_{i}(x)$. The fact that there is no moduli that change the size of the solitons has been known from the analysis of the massless modes of the open string connecting D0 branes and D2 branes, but one may have expected that the soliton has a fixed size set by the noncommutative parameter $\theta^{i j}$. This turned out not to be the case for these solutions. There are various other solutions, describing branes intersecting with each other with arbitrary angles, which can be expressed in the form (1.2), and they all have delta-function singularities after the Seiberg-Witten transform.

On the other hand, solutions in higher dimensions are not necessarily of the form (1.2) and therefore can have a finite size after the Seiberg-Witten transform. We examine in detail the $U(2)$ instanton constructed in Ref. [16]. The solution contains an extra modulus $\rho$, which in the commutative limit $\theta \rightarrow 0$ reduces to the size of the instanton. We evaluate the Seiberg-Witten transform of this solution in the two limit, $\rho \ll \sqrt{\theta}$ and $\sqrt{\theta} \ll \rho$. When $\rho=0$, the instanton solution is of the form (1.2) and has a delta-function singularity when expressed in the commutative variables. We find that, as soon as we turn on a small amount of $\rho$, the solution gets a nonzero support of the size $\sim \sqrt{\theta}$. We also see that the deltafunction singularity is modified by $\rho$. On the other hand, for $\sqrt{\theta} \ll \rho$, we find that the delta-function singularity is completely resolved and that the solution has a smooth profile, which, for the first two terms in the $1 / \rho$ expansion, precisely agrees with that of the Belavin-Polyakov-Schwarz-Tyupkin (BPST) instanton on commutative space.

This paper is organized as follows. In Sec. II, we review the construction of the Seiberg-Witten map derived in [4648]. In Sec. III, we evaluate Seiberg-Witten transform of the noncommutative solitons in $(2+1)$ dimensions, which take the form (1.2). Other examples, including intersecting branes and fluxons, are discussed in Sec. IV. In Sec. V, we study the Seiberg-Witten transform of the $U(2)$ noncommutative in- stanton solution and show how the delta-function singularity is resolved. We will close this paper with discussions of our results in Sec. VI. In the Appendices, we derive some of the formulas used in this paper and give some details of the computation in Sec. V.

\section{SEIBERG-WITTEN MAP}

In Refs. [46-48], an exact and explicit form of the Seiberg-Witten map for the $U(1)$ part of the field strength was obtained from string theory computation of the coupling between the noncommutative gauge theory on the branes and the Ramond-Ramond potentials in the bulk. For a gauge theory with $2 n$ noncommutative dimensions, the map from the field strength in the noncommutative variables $\hat{A}_{i}$

$$
\hat{F}_{i j}=\partial_{i} \hat{A}_{j}-\partial_{j} \hat{A}_{i}+i \hat{A}_{i} * \hat{A}_{j}-i \hat{A}_{j} * \hat{A}_{i}
$$

to the field strength $F_{i j}=\partial_{i} A_{j}-\partial_{j} A_{i}$ of the commutative variables $A_{i}$, is given ${ }^{3}$ in the Fourier transformed form by

$$
\begin{aligned}
F_{i j}(k) & -\theta_{i j}^{-1} \delta(k) \\
= & \frac{1}{P f(\theta)} \int d x *\left\{e^{i k \cdot X}(\theta+\theta \hat{f} \theta)_{i j}^{n-1} P\right. \\
& \left.\times \exp \left[i \int_{0}^{1} \hat{A}_{i}(\hat{x}+l r) l^{i} d \tau\right]\right\},
\end{aligned}
$$

where

$$
\begin{aligned}
(\theta+\theta \hat{f} \theta)_{i j}^{n-1}= & \frac{1}{2^{n-1}(n-1) !} \epsilon_{i j i_{1} i_{2} \cdots i_{2 n-2}} \\
& \times \int_{0}^{1} d \tau_{1}\left[\theta+\theta \hat{F}\left(\hat{x}+l \tau_{1}\right) \theta\right]^{i_{1} i_{2} \ldots} \\
& \times \int_{0}^{1} d \tau_{n-1}[\theta+\theta \hat{F}(\hat{x} \\
& \left.\left.+l \tau_{n-1}\right) \theta\right]^{i_{2 n-3}-i_{2 n-2}} .
\end{aligned}
$$

In particular, for $n=1$ and 2, we have

$$
\begin{aligned}
&(\theta+\theta \hat{f} \theta)_{i j}^{n-1} \\
& \quad= \begin{cases}\epsilon_{i j} & (n=1) \\
\frac{1}{2} \epsilon_{i j k l} \int_{0}^{1} d \tau[\theta+\theta \hat{F}(\hat{x}+l \tau) \theta]^{k l} & (n=2) .\end{cases}
\end{aligned}
$$

\footnotetext{
${ }^{3}$ In this paper, we choose the sign of the noncommutative parameter $\theta^{i j}$ as in Eq. (1.1). To use the convention in Ref. [46], one can simply make the substitution $\theta^{i j} \rightarrow-\theta^{i j}$ in the following.
} 
This expression involves the open Wilson line, which is a basic building block of observables in noncommutative gauge theory $[59,60,50,24]$. In order to actually evaluate the Seiberg-Witten map, it is useful to express it using the variable $X^{i}$ defined by Eq. (1.3). For $n=1$, the Seiberg-Witten map is given by

$$
F_{12}(k)-\theta_{12}^{-1} \delta(k)=\operatorname{Tr} e^{i k \cdot X}
$$

and for $n=2$ by

$$
F_{i j}(k)-\theta_{i j}^{-1} \delta(k)=-\frac{i}{2} \epsilon_{i j k l} \operatorname{Tr}\left(\left[X^{k}, X^{l}\right] e^{i k \cdot X}\right) .
$$

When the noncommutative gauge theory is realized on $\mathrm{D} p$ branes, the field strength $F_{i j}(k)$ of the commutative variables $A_{i}(x)$ can be regarded as the $\mathrm{D}(p-2)$ brane density on the $\mathrm{D} p$ branes. This was how the expression (2.2) was found in [46-48]. In the following, we will find it useful to consider lower brane densities also. The $\mathrm{D}(p-2 s)$ brane density on the $\mathrm{D} p$ branes is given by

$$
\begin{aligned}
J_{i_{1} \cdots i_{p-2 s}} \sim & \epsilon_{i_{1} \cdots i_{p-2 s} j_{1} \cdots j_{2 s}} \int_{0}^{1} d \tau_{1} \int_{\tau_{1}}^{1} d \tau_{2} \cdots \int_{\tau_{s-2}}^{1} d \tau_{s-1} \\
& \times \operatorname{Tr}\left(\left[X^{j_{1}}, X^{j_{2}}\right] e^{i \tau_{1} k \cdot X}\left[X^{j_{3}}, X^{j_{4}}\right] e^{i\left(\tau_{2}-\tau_{1}\right) k \cdot X} \cdots\left[X^{j_{2 s}-1}, X^{j_{2 s}}\right] e^{i\left(1-\tau_{s-1}\right) k \cdot X}\right) .
\end{aligned}
$$

\section{SOLITONS IN 2+1 DIMENSIONS}

In Ref. [15], all static classical solutions to the noncommutative Yang-Mills theory in $(2+1)$ dimensions are classified. They take the form

$$
X^{i}=U \hat{x}^{i} U^{\dagger}+\sum_{a=1}^{m} \lambda_{a}^{i}|a\rangle\langle a| \quad(i=1,2),
$$

where $\{|a\rangle\}_{a=1 \cdots m}$ is an $m$-dimensional subspace of the Fock space of Eq. (1.1) times $\mathbf{C}^{N}, \lambda_{a}^{i}$ 's are arbitrary constant parameters, and $U$ is the associated shift operator obeying

$$
U^{\dagger} U=1, \quad U U^{\dagger}=1-\sum_{a=1}^{m}|a\rangle\langle a| .
$$

It is straightforward to compute the Seiberg-Witten transform of this solution. ${ }^{4}$

Substituting Eq. (3.1) into the Seiberg-Witten map (2.5), we find

$$
\begin{aligned}
\operatorname{tr} e^{i k \cdot X} & =\operatorname{tr}\left[U e^{i k \cdot \hat{x}} U^{\dagger}+\sum_{a=0}^{m-1} e^{i k_{1} \lambda_{a}^{i}}|a\rangle\langle a|\right] \\
& =\operatorname{tr} e^{i k \cdot \hat{x}}+\sum_{a=0}^{m-1} e^{i k_{i} \lambda_{a}^{i}}\langle a \mid a\rangle \\
& =\frac{1}{\theta} \delta(k)+\sum_{a=0}^{m-1} e^{i k_{i} \lambda_{a}^{i}} .
\end{aligned}
$$

\footnotetext{
${ }^{4}$ This is essentially the same as the computation of the Wilson line observables in the soliton background discussed in Refs. [15,50]. Here we are reinterpreting it as an evaluation of the Seiberg-Witten map.
}

Here in the first equality we have used the following identity:

$$
e^{i U k \cdot \hat{x} U^{\dagger}}=U e^{i k \cdot \hat{x}} U^{\dagger}+1-U U^{\dagger}
$$

The field strength expressed in the commutative variables is then $^{5}$

$$
F_{12}(k)=\sum_{a=0}^{m-1} e^{i k_{i} \lambda_{a}^{i}}
$$

By taking the Fourier transform of this, we find

$$
F_{12}(x)=\sum_{a=0}^{m-1} \delta\left(x-\lambda_{a}\right) .
$$

We see that the solution has delta-function supports at $x$ $=\lambda_{a}(a=0, \ldots, m-1)$. This gives a precise interpretation of the moduli $\lambda_{a}$ as representing the locations of the soliton, confirming the observations in Refs. $[11,15,50]$.

There is an obvious generalization of this construction to higher dimensions. Let us assume that $\theta^{12}, \theta^{34}, \ldots, \theta^{i_{2 n-1} i_{2 n}}$ $\neq 0$ and other $=0$ so that we have a direct product of $n$ Fock spaces. We can then consider a solution

$$
X^{i}=U x^{i} U^{\dagger}, \quad(i=1, \ldots, 2 n) .
$$

Here we set all the moduli $\lambda=0$ for simplicity, and $U$ is a shift operator of rank $m$. The Seiberg-Witten map in $2 n$ dimensions is

\footnotetext{
${ }^{5}$ Note that, since $\theta^{i j}$ is antisymmetric, $\theta_{12}^{-1}=-1 / \theta^{12}$.
} 


$$
\begin{aligned}
F_{i j}(k)-\theta_{i j}^{-1} \delta(k)= & -\frac{i^{n-1}}{2^{n-1}(n-1)} \epsilon_{i j s_{1} \cdots s_{2 n-2}} \int_{0}^{1} d \tau_{1} \cdots \int_{\tau_{n-3}}^{1} d \tau_{n-2} \\
& \times \operatorname{tr}\left(\left[X^{s_{1}}, X^{s_{2}}\right] e^{i \tau_{1} k \cdot X} \cdot\left[X^{s_{2 n-3}}, X^{s_{2 n-2}}\right] e^{i\left(1-\tau_{n-2}\right) k \cdot X}\right) .
\end{aligned}
$$

Using Eq. (3.4) and

$$
\left[X^{i}, X^{j}\right]=i U \theta^{i j} U^{\dagger},
$$

one finds that the right-hand side of Eq. (3.8) is $-\theta_{i j}^{-1} \delta(k)$, and therefore

$$
F_{i j}(k)=0 \text {. }
$$

Similarly one can show that the soliton does not give a nontrivial contribution to the the $\mathrm{D} 2 p$ brane density for all $p$ $\geqslant 1$. The only nonvanishing one is the D0 brane density, which is given by

$$
\begin{aligned}
J(k) & =\operatorname{Tr}\left(e^{i k \cdot X}\right) \\
& =\operatorname{Tr}\left(U e^{i k \cdot \hat{x}} U^{\dagger}+1-U U^{\dagger}\right) \\
& =\frac{1}{\operatorname{Pf}(\theta)} \delta(k)+m .
\end{aligned}
$$

The Fourier transform of this gives

$$
J(x)=\frac{1}{\operatorname{Pf}(\theta)}+m \delta(x) .
$$

The first term represents the background D0 brane charge in the presence of the constant $B$ field and the second term corresponds to the $m$ D0 branes described by the soliton solution (3.7). This soliton therefore describes $m$ D0 branes without higher brane charges.

One may be puzzled by that fact that the solution (3.7) of the noncommutative $U(1)$ gauge theory describes D0 branes, even though the field strength $F_{i j}$ of this solution is identically equal to zero. Such a bizarre behavior is not unexpected for solutions with delta-function singularities. To illustrate the point, let us imagine that $F_{i j}$ has the following configuration:

$$
F_{i j} \sim \epsilon^{-2} \exp \left(-\frac{x^{2}}{\epsilon^{2}}\right)
$$

In this case,

$$
\epsilon^{i_{1} \cdots i_{2 n}} F_{i_{1} i_{2}} \cdots F_{i_{2 n-1} i_{2 n}} \sim \epsilon^{-2 n} \exp \left(-n \frac{x^{2}}{\epsilon^{2}}\right) .
$$

In the limit $\epsilon \rightarrow 0$, the field strength vanishes $F_{i j} \rightarrow 0$, but $F^{n}$ becomes proportional to $\delta(x)$.

If we embed the solution (3.7) to the $U(N)$ gauge theory, it is possible to deform it away from the form (3.7). In Sec. $\mathrm{V}$, we study the $U(2)$ instanton solution in four dimensions, for which an explicit expression is known [16]. We find that the Seiberg-Witten transform of the instanton acquires a finite size as soon as we turn on the deformation, and the size is set by the noncommutative scale $\theta$ and the deformation parameter $\rho$. We also show the $U(1)$ part of the field strength becomes nonzero after the deformation.

\section{INTERSECTING BRANES}

Noncommutative soliton solutions representing orthogonally intersecting branes have been constructed in literature $[29,30]$. In this section, we generalize these constructions by allowing arbitrary angles and evaluate their Seiberg-Witten transforms.

\section{A. D2 branes orthogonally intersecting on a D4 brane}

As a warm up, let us consider D2 branes orthogonally intersecting on a D4 brane world volume. It can be obtained by reinterpreting the tachyon configuration studied in Ref. [29] as a gauge field configuration on the D4 brane:

$$
X^{1,2}=U \hat{x}^{1,2} U^{\dagger} \otimes 1, \quad X^{3,4}=1 \otimes V \hat{x}^{3,4} V^{\dagger} .
$$

Here we introduced noncommutativity as $\theta^{12}, \theta^{34} \neq 0$, and so we have a direct product of the two Fock spaces. The operator $V$ is the same as $U$ except that $V$ acts on the second Fock space of $\hat{x}^{3}$ and $\hat{x}^{4}$ :

$$
U \equiv \sum_{n}|n+m\rangle\left\langle n\left|\otimes 1, \quad V \equiv 1 \otimes \sum_{n}\right| n+l\right\rangle\langle n|
$$

The above solution represents the brane configuration in which $m$ D2 branes localized at the origin of the $x^{1}-x^{2}$ plane are intersecting with $l$ D2 branes localized at the origin of the $x^{3}-x^{4}$ plane. This geometrical interpretation is confirmed by evaluating the Seiberg-Witten map (2.6) for four noncommutative dimensions:

$$
F_{12}(x)=m \delta\left(x^{1}\right) \delta\left(x^{2}\right), \quad F_{34}(x)=l \delta\left(x^{3}\right) \delta\left(x^{4}\right), \quad \text { others }=0 .
$$

It is also interesting to calculate the D0-brane density using Eq. (2.7) with $p=4, s=2$ :

$$
\begin{aligned}
J(k)= & \operatorname{Tr}\left(e^{i k \cdot X}\right) \\
= & \operatorname{Tr}\left[U \exp \left(i k_{1} \hat{x}^{1}+i k_{2} \hat{x}^{2}\right) U^{\dagger} \otimes V\right. \\
& \times \exp \left(i k_{3} \hat{x}^{3}+i k_{4} \hat{x}^{4}\right) V^{\dagger}+\sum_{a=0}^{m-1}|a\rangle\langle a| \otimes V \\
& \times \exp \left(i k_{3} \hat{x}^{3}+i k_{4} \hat{x}^{4}\right) V^{\dagger}+U \exp \left(i k_{1} \hat{x}^{1}+i k_{2} \hat{x}^{2}\right) U^{\dagger}
\end{aligned}
$$




$$
\begin{aligned}
& \left.\otimes \sum_{a=0}^{l-1}|a\rangle\left\langle a\left|+\sum_{a=0}^{m-1}\right| a\right\rangle\left\langle a\left|\otimes \sum_{a=0}^{l-1}\right| a\right\rangle\langle a|\right] \\
= & \frac{1}{\theta^{12} \theta^{34}} \delta^{4}(k)+\frac{m}{\theta^{34}} \delta\left(k_{3}\right) \delta\left(k_{4}\right) \\
& +\frac{l}{\theta^{12}} \delta\left(k_{1}\right) \delta\left(k_{2}\right)+m l .
\end{aligned}
$$

After the Fourier transformation, we obtain

$$
\begin{aligned}
J(x)= & \frac{1}{\theta^{12} \theta^{34}}+\frac{m}{\theta^{34}} \delta\left(x^{1}\right) \delta\left(x^{2}\right)+\frac{l}{\theta^{12}} \delta\left(x^{3}\right) \delta\left(x^{4}\right) \\
& +m l \delta^{4}(x) .
\end{aligned}
$$

It is interesting to note that, using Eq. (4.3), this can be expressed as

$$
J(x)=\frac{1}{8} \epsilon^{i j k l}\left[F_{i j}(x)-\theta_{i j}^{-1}\right]\left[F_{k l}(x)-\theta_{k l}^{-1}\right] .
$$

Such a relation between the D0-brane charge density $J(x)$ and the field strength $F_{i j}$ holds in the leading order in the standard $\alpha^{\prime}$ expansion of string theory computation, but it is expected to receive large corrections in the Seiberg-Witten limit. In fact, in the more elaborate examples discussed below, such a relation does not hold.

\section{B. Intersection with arbitrary angles}

We can introduce an arbitrary angle to the solution (4.1) by deforming it as follows:

$$
\begin{aligned}
& X^{1,2}=U \hat{x}^{1,2} U^{\dagger} \otimes 1+\sum_{a=0}^{m-1}|a\rangle\langle a| \otimes \lambda_{a}^{1,2}\left(\hat{x}^{3}, \hat{x}^{4}\right), \\
& X^{3,4}=1 \otimes \hat{x}^{3,4},
\end{aligned}
$$

where $\lambda$ 's are functions of $\hat{x}^{3}$ and $\hat{x}^{4}$. Here we have set $l$ $=0$ so that the configuration does not include localized D0 branes [see the last term in Eq. (4.5)]. Substituting this into the equation of motion,

$$
\left(X_{i},\left[X^{i}, X^{j}\right]\right)=0
$$

we find that $\lambda$ 's have to be linear functions,

$$
\lambda_{a}^{i}\left(\hat{x}^{3}, \hat{x}^{4}\right)=\alpha_{a}^{i}+\beta_{a}^{i} \hat{x}^{3}+\gamma_{a}^{i} \hat{x}^{4},
$$

where $\alpha, \beta$, and $\gamma$ are constant parameters, and $i=1,2$.

We can regard $\lambda_{a}$ 's as representing the configurations of the D2 branes. To confirm this interpretation, we evaluate the Seiberg-Witten map (2.6):

$$
\begin{aligned}
& F_{12}(x)=\sum_{a=0}^{m-1} \delta_{a}(x), \quad F_{34}(x)=\sum_{a=0}^{m-1}\left(\beta_{a}^{1} \gamma_{a}^{2}-\beta_{a}^{2} \gamma_{a}^{1}\right) \delta_{a}(x), \\
& F_{13}(x)=-\sum_{a=0}^{m-1} \beta_{a}^{2} \delta_{a}(x), \quad F_{23}(x)=\sum_{a=0}^{m-1} \beta_{a}^{1} \delta_{a}(x),
\end{aligned}
$$

$$
F_{14}(x)=-\sum_{a=0}^{m-1} \gamma_{a}^{2} \delta_{a}(x), \quad F_{24}(x)=\sum_{a=0}^{m-1} \gamma_{a}^{1} \delta_{a}(x),
$$

where

$$
\delta_{a}(x) \equiv \delta\left[x^{1}-\lambda_{a}^{1}\left(x^{3}, x^{4}\right)\right] \delta\left[x^{2}-\lambda_{a}^{2}\left(x^{3}, x^{4}\right)\right] .
$$

Therefore the D2 branes are located as expected. It is also useful to point out that Eq. (4.11) satisfies the Bianchi identity, $\partial_{[i,} F_{j, k]}=0$. For example,

$$
\begin{aligned}
\partial_{\left[1, F_{2,3]}\right.} & =\sum_{a}\left(\partial_{3}+\beta_{a}^{1} \partial_{1}+\beta_{a}^{2} \partial_{2}\right) \delta\left(x^{1}-\lambda_{a}^{1}\right) \delta\left(x^{2}-\lambda_{a}^{2}\right) \\
& =0
\end{aligned}
$$

consistently with the general proof in Ref. [46].

The D0-brane density for this solution is

$$
\begin{aligned}
J(k)= & \operatorname{Tr}\left[U \exp \left(i k_{1} \hat{x}^{1}+i k_{2} \hat{x}^{2}\right) U^{\dagger} \otimes \exp \left(i k_{3} \hat{x}^{3}+i k_{4} \hat{x}^{4}\right)\right. \\
& \left.+\sum_{a=0}^{m-1}|a\rangle\langle a| \otimes \exp \left(i k_{1} \lambda_{a}^{1}+i k_{2} \lambda_{a}^{2}+i k_{3} \hat{x}^{3}+i k_{4} \hat{x}^{4}\right)\right] \\
= & \frac{1}{\operatorname{Pf}(\theta)} \delta^{4}(k)+\frac{1}{\theta^{34}} \sum_{a} e^{i k_{a} \alpha_{a}^{1}+i k_{2} \alpha_{a}^{2}} \\
& \times \delta\left(k_{1} \beta_{a}^{1}+k_{2} \beta_{a}^{2}+k_{3}\right) \delta\left(k_{1} \gamma_{a}^{1}+k_{2} \gamma_{a}^{2}+k_{4}\right) .
\end{aligned}
$$

After performing the Fourier transformation, we obtain

$$
J(x)=\frac{1}{\operatorname{Pf}(\theta)}+\frac{1}{\theta^{34}} \sum_{a=0}^{m-1} \delta_{a}(x) .
$$

As before, the first term shows the uniform distribution of the D0 branes in the D4 brane. The second term indicates the D0 branes bound in the D2 branes located at the place where the delta functions specify. There is no localized D0 brane in this case.

We have shown that it is possible to introduce moduli to the intersecting brane solutions as in Eqs. (4.7) and (4.8) to describe configurations of branes with arbitrary angles. We can generalize this further by introducing additional moduli as

$$
\begin{aligned}
X^{1,2}= & U \hat{x}^{1,2} U^{\dagger} \otimes 1+\sum_{a=0}^{m-1}|a\rangle\langle a| \otimes \lambda_{a}^{1,2}\left(\hat{x}^{3}, \hat{x}^{4}\right) \\
& +\sum_{a, b} \zeta_{a b}^{1,2}|a\rangle\langle a|\otimes| b\rangle\langle b|, \\
X^{3,4}= & 1 \otimes V \hat{x}^{3,4} V^{\dagger}+\sum_{b=0}^{l-1} \lambda_{b}^{3,4}\left(\hat{x}^{1}, \hat{x}^{2}\right) \otimes|a\rangle\langle a| \\
& +\sum_{a, b} \zeta_{a b}^{3,4}|a\rangle\langle a|\otimes| b\rangle\langle b| .
\end{aligned}
$$


The Seiberg-Witten map gives

$$
\begin{aligned}
F_{12}(x)= & \sum_{a=0}^{m-1} \delta\left[x^{1}-\lambda_{a}^{1}\left(x^{3}, x^{4}\right)\right] \delta\left[x^{2}-\lambda_{a}^{2}\left(x^{3}, x^{4}\right)\right] \\
& +\sum_{b=0}^{l-1}\left(\beta_{b}^{3} \gamma_{b}^{4}-\beta_{b}^{4} \gamma_{b}^{3}\right) \delta\left[x^{3}-\lambda_{a}^{3}\left(x^{1}, x^{2}\right)\right] \\
& \times \delta\left[x^{4}-\lambda_{a}^{4}\left(x^{1}, x^{2}\right)\right],
\end{aligned}
$$

and similar expressions for the other components of the gauge field strength. Note that the number of the D0 branes is $m l$, whereas the number of the $\mathrm{D} 2$ branes is $m+l$. The $\mathrm{D} 0$ brane density is given by

$$
\begin{aligned}
J(x)= & \frac{1}{\operatorname{Pf}(\theta)}+\frac{1}{\theta} \sum_{a=0}^{m-1} \delta\left[x^{1}-\lambda_{a}^{1}\left(x^{3}, x^{4}\right)\right] \delta\left[x^{2}-\lambda_{a}^{2}\left(x^{3}, x^{4}\right)\right] \\
& +\frac{1}{\theta^{12}} \sum_{b=0}^{l-1} \delta\left[x^{3}-\lambda_{a}^{3}\left(x^{1}, x^{2}\right)\right] \delta\left[x^{4}-\lambda_{a}^{4}\left(x^{1}, x^{2}\right)\right] \\
& +\sum_{a, b} \Pi_{i=1}^{4} \delta\left(x^{i}-\zeta_{a b}^{i}\right) .
\end{aligned}
$$

The last term shows the localized D0 branes scattered in the D4 brane.

It is straightforward to include the scalar field in this construction and allow the D2 branes and the D0 branes to move away from the D4 brane, as discussed in Ref. [11].

\section{D1 branes intersecting with the D3 brane}

The solutions discussed so far are all non-BPS and unstable. One of the interesting BPS noncommutative solutions is the fluxon solution studied in $[8,15,17,18]$. If we turn on the noncommutativity only along the $x^{1}-x^{2}$ plane, the solution representing D1 branes piercing a D3 brane is

$$
\begin{aligned}
& X^{i}=U \hat{x}^{i} U^{\dagger}+\sum_{a=0}^{m-1}|a\rangle\langle a| \lambda_{a}^{i}, \\
& A_{3}=0, \quad \hat{\Phi}=\frac{1}{\theta^{12}} \sum_{a=0}^{m-1}\left(x_{3}-\zeta_{a}\right)|a\rangle\langle a| .
\end{aligned}
$$

Note that $i=1,2$ and there is no noncommutativity along $x^{3}$. The above solution satisfies the BPS equations in noncommutative Yang-Mills theory on the D3 brane,

$$
\begin{gathered}
-\partial_{3} \hat{\Phi}=B_{3} \equiv \frac{-i}{\left(\theta^{12}\right)^{2}}\left(\left[X^{1}, X^{2}\right]-i \theta^{12}\right), \\
i\left[X^{1}, \hat{\Phi}\right] / \theta^{12}=\hat{B}_{2}, \quad-i\left[X^{2}, \hat{\Phi}\right] / \theta^{12}=\hat{B}_{1} .
\end{gathered}
$$

The last two equations are trivially satisfied since both sides of the two equations vanish.

The D1-brane current density of this solution is

$$
\begin{aligned}
\operatorname{Tr} \exp \left(i k_{1} X^{1}+i k_{2} X^{2}+i k_{\Phi} \Phi\right) \\
=\frac{1}{\theta^{12}} \delta\left(k_{1}\right) \delta\left(k_{2}\right)+\sum_{a} \exp \left[i k_{1} \lambda_{a}^{1}+i k_{2} \lambda_{a}^{2}\right. \\
\left.\quad+i k_{\Phi} \frac{1}{\theta^{12}}\left(x_{3}-\zeta_{a}\right)\right],
\end{aligned}
$$

where note that we have introduced a transverse momentum $k_{\Phi}$ coupled to $\Phi$. The Fourier transform of this expression is

$$
\begin{gathered}
J(x)=\int d k_{1} d k_{2} d k_{\Phi} e^{-i k_{1} x^{1}-i k_{2} x^{2}-i k_{\Phi} \Phi} J(k) \\
=\frac{1}{\theta^{12}} \delta(\Phi)+\sum_{a} \delta\left(x^{1}-\lambda_{a}^{1}\right) \delta\left(x^{2}-\lambda_{a}^{2}\right) \\
\quad \times \delta\left[\Phi-\left(x_{3}-\zeta_{a}\right) / \theta^{12}\right] .
\end{gathered}
$$

The first term shows the D1 branes uniformly distributed on the D3 brane as a result of the background B-field $B_{12}$. The second term shows the D1 branes intersecting with the D3 brane. We note that the intersection angle depends on $\theta$, as expected for the BPS solution. The intersection point is located at $\left(\lambda_{a}^{1}, \lambda_{a}^{2}, \zeta_{a}\right)$ on the world volume of the D3 brane.

It is easy to generalize this solution to various other cases, e.g., infinite number of D1 branes piercing Ref. [18], introducing another transverse scalar field in such a way that the D1 brane is completely apart from the D3 brane [17], and non-BPS deformation by changing the tilt of the D1 brane [15]. The Seiberg-Witten transforms of these solutions confirm the known interpretations of these solitons and their moduli.

\section{INSTANTONS AND RESOLUTION OF THE DELTA FUNCTION SINGULARITIES}

We have found that solutions constructed using projection operators have delta-function singularities. In this section, we will study how these singularities are resolved in the case of the $U(2)$ instanton solution on the four dimensional noncommutative space with a single scale modulus $\rho$.

For definiteness, we assume that the noncommutative parameter $\theta^{i j}$ is anti-self-dual and set

$$
\theta^{34}=-\theta^{12}=\theta>0, \text { other }=0 .
$$

Given this, there is a distinction between self-dual and antiself-dual solutions, constructed in Refs. [2,4] and in Ref. [16] respectively. In this section, we examine the anti-self-dual solution of Ref. [16] since it can be regarded as a deformation of a solution of the form (1.2) embedded in the $U(2)$ theory, as we will see explicitly in Eqs. (5.6) and (5.7).

Let us review the construction of the anti-self-dual solution in Ref. [16]. To simplify the computations in the following, we rescale the coordinates $\hat{x}^{i}$ so that the noncommutative scale is set as $\theta=1$. Whenever necessary, we can restore $\theta$ by a simple dimensional analysis. It is useful to combine the coordinates into the form of the creation and annihilation operators 


$$
a_{1} \equiv \frac{1}{\sqrt{2}}\left(\hat{x}^{2}+i \hat{x}^{1}\right), \quad a_{2} \equiv \frac{1}{\sqrt{2}}\left(\hat{x}^{4}-i \hat{x}^{3}\right)
$$

satisfying the standard commutation relation,

$$
\left[a_{i}, a_{j}^{\dagger}\right]=\delta_{i j}
$$

and acting on the Fock space $\{|n, m\rangle \mid n, m \geqslant 0, \in \mathbf{Z}\}$. Using this notation, the $U(2)$ anti-self-dual instanton solution $X^{\mu}$ is expressed as

$$
X^{\mu}=\Psi^{\dagger} \hat{x}^{\mu} \Psi=U^{\dagger} \hat{x}^{\mu} U+\rho^{2}\left(\begin{array}{cc}
{\left[2(N+2)+\rho^{2}\right]^{-1 / 2} \hat{x}^{\mu}\left[2(N+2)+\rho^{2}\right]^{-1 / 2}} & 0 \\
0 & \left(2 N+\rho^{2}\right)^{-1 / 2} \hat{x}^{\mu}\left(2 N+\rho^{2}\right)^{-1 / 2}
\end{array}\right)
$$

where

$$
\Psi=\left(\Psi^{(1)}, \Psi^{(2)}\right), \quad \Psi^{(1)}=\left(\begin{array}{c}
\rho \\
0 \\
\sqrt{2} a_{2}^{\dagger} \\
-\sqrt{2} a_{1}^{\dagger}
\end{array}\right) \frac{1}{\sqrt{2(N+2)+\rho^{2}}}
$$

$\Psi^{(2)}=\left(\begin{array}{c}0 \\ \rho \\ \sqrt{2} a_{1} \\ \sqrt{2} a_{2}\end{array}\right) \frac{1}{\sqrt{2 N+\rho^{2}}}$

and thus

$$
U \equiv\left(N+2+\frac{\rho^{2}}{2}\right)^{-1 / 2}\left(\begin{array}{cc}
a_{2}^{\dagger} & a_{1} \\
-a_{1}^{\dagger} & a_{2}
\end{array}\right)
$$

Here $N$ is the number operator $N \equiv a_{1}^{\dagger} a_{1}+a_{2}^{\dagger} a_{2}$ and $\rho$ is a parameter of the solution, which is related to the size of the solution as we will see below. In the following, when we restore $\theta$, we assign the dimension of length to the parameter $\rho$.

In the limit of $\rho \rightarrow 0$, the solution (5.4) becomes the zero size instanton of the form (3.1), as discussed in Ref. [16]. To see this, we note that the second term in Eq. (5.4) disappears in this limit, and the solution becomes

$$
X^{\mu}=U_{0}^{\dagger} \hat{x}^{\mu} U_{0},
$$

where the operator $\left.U_{0} \equiv U\right|_{\rho=0}$ satisfies $^{6}$

$$
U_{0} U_{0}^{\dagger}=\left(\begin{array}{cc}
1 & 0 \\
0 & 1
\end{array}\right), \quad U_{0}^{\dagger} U_{0}=\left(\begin{array}{cc}
1 & 0 \\
0 & 1-|0,0\rangle\langle 0,0|
\end{array}\right) .
$$

\footnotetext{
${ }^{6}$ Note that, compared with the construction in the previous sections, the roles of $U_{0}$ and $U_{0}^{\dagger}$ are exchanged. In this section, we are following the notations of Ref. [16].
}

Therefore this $U_{0}$ can be regarded as a shift operator, and the Seiberg-Witten transform can be evaluated in the same way as in the previous sections. For example, the D0 brane density is given by

$$
J(k)=\operatorname{Tr} e^{i k \cdot X}=2 \delta(k)+1,
$$

or in the $x$ space by

$$
J(x)=\frac{2}{\theta^{2}}+\delta(x)
$$

(Here we have restored $\theta$.) The first term is for the uniform distribution of the D0 branes on the parallel two D4 branes, and the second term gives the localized additional D0 brane charge.

Now we consider the resolution of this singularity by turning on the modulus $\rho$. In the following, we distinguish the three types of traces: $\operatorname{tr}(\cdots)$ is over the $U(2)$ group indices, $\operatorname{Tr}(\cdots)$ is over the Fock space, and the combined trace is expressed as $\mathbf{T r}=\operatorname{tr} T r$.

\section{A. Small $\rho$ expansion}

Let us first turn on a small value of $\rho$ and see what happens. The solution (5.4) can be expanded in powers of $\rho$ as

$$
\Psi^{\dagger} k \cdot \hat{x} \Psi=A+\frac{\rho}{\sqrt{2}}(B+C)+\frac{\rho^{2}}{2}(D+E+F+G)+\mathcal{O}\left(\rho^{3}\right),
$$

where

$$
A \equiv U_{0}^{\dagger} k \cdot \hat{x} U_{0}
$$

$$
B \equiv k \cdot \hat{x}|0,0\rangle\langle 0,0| \otimes\left(\begin{array}{cc}
0 & 0 \\
0 & 1
\end{array}\right)
$$

$$
C \equiv|0,0\rangle\langle 0,0| k \cdot \hat{x} \otimes\left(\begin{array}{ll}
0 & 0 \\
0 & 1
\end{array}\right)
$$




$$
\begin{aligned}
& D \equiv \frac{1}{\sqrt{N_{\neq 0}}} k \cdot \hat{x} \frac{1}{\sqrt{N_{\neq 0}}} \otimes\left(\begin{array}{ll}
0 & 0 \\
0 & 1
\end{array}\right), \\
& E \equiv \frac{1}{\sqrt{N+2}} k \cdot \hat{x} \frac{1}{\sqrt{N+2}} \otimes\left(\begin{array}{ll}
1 & 0 \\
0 & 0
\end{array}\right), \\
& F \equiv-\frac{1}{2} U_{0}^{\dagger} \frac{1}{N+1} k \cdot \hat{x} U_{0}, \\
& G \equiv-\frac{1}{2} U_{0}^{\dagger} k \cdot \hat{x} \frac{1}{N+1} U_{0} .
\end{aligned}
$$

The operator $1 / N_{\neq 0}$ is defined in the projected Fock space $\{(1-|0,0\rangle\langle 0,0|)|n, m\rangle\}$. Let us examine the D0-brane density of the solution expanding again in powers of $\rho$,

$$
\begin{aligned}
J(k) \equiv & \operatorname{Tr}\left[\exp \left(i \Psi^{\dagger} k \cdot \hat{x} \Psi\right)\right] \\
= & \operatorname{Tr}\left(e^{i A}\right)-\frac{\rho^{2}}{2} \int_{0}^{1} d \tau \operatorname{Tr}\left(C e^{i \tau A} B\right) \\
& +\frac{\rho^{2}}{2} i \operatorname{Tr}\left[(D+E+F+G) e^{i A}\right]+\mathcal{O}\left(\rho^{4}\right) .
\end{aligned}
$$

Here we used relations

$$
A C=B A=B^{2}=C^{2}=0 .
$$

As expected, the first term in the right-hand side of Eq. (5.15) reproduces Eq. (5.8):

$$
\operatorname{Tr}\left(e^{i A}\right)=2 \delta(k)+1
$$

Now we are going to evaluate the second term of the right-hand side in Eq. (5.15). Using the relation (3.4), we obtain

$$
\begin{aligned}
\operatorname{Tr}[ & \left.C e^{i \tau k \cdot \hat{x}} B\right] \\
& =\operatorname{tr}\left\langle 0,0\left|k \cdot \hat{x}\left(\begin{array}{cc}
0 & 0 \\
0 & 1
\end{array}\right) U_{0}^{\dagger} e^{i \tau k \cdot \hat{x}} U_{0}\left(\begin{array}{cc}
0 & 0 \\
0 & 1
\end{array}\right) k \cdot \hat{x}\right| 0,0\right\rangle \\
& =\frac{k^{2}}{2}\left\langle 0,0\left|e^{i \tau k \cdot \hat{x}}\right| 0,0\right\rangle \\
& =\frac{k^{2}}{2} e^{-\tau^{2} k^{2} / 4}
\end{aligned}
$$

Therefore the second term in Eq. (5.15) can be written as

$$
-\frac{\rho^{2}}{2} \int_{0}^{1} d \tau \operatorname{Tr}\left(C e^{i \tau A} B\right)=-\frac{1}{4} k^{2} \rho^{2} \int_{0}^{1} d \tau e^{-\tau^{2} k^{2} / 4} .
$$

Let us proceed to the third term of the right-hand side of Eq. (5.15). First, we note

$$
\begin{aligned}
\operatorname{Tr}\left[D e^{i A}\right]= & \operatorname{Tr}\left[U_{0} D U_{0}^{\dagger} e^{i k \cdot \hat{x}}\right] \\
= & \operatorname{Tr}\left[\frac{1}{N+1}\left(a_{1} k \cdot \hat{x} a_{1}^{\dagger}+a_{2} k \cdot \hat{x} a_{2}^{\dagger}\right) \frac{1}{N+1} e^{i k \cdot \hat{x}}\right] \\
= & \operatorname{Tr}\left[\frac{1}{N+1} k \cdot \hat{x} \frac{N+2}{N+1} e^{i k \cdot \hat{x}}\right] \\
& +\frac{1}{\sqrt{2}} \operatorname{Tr}\left[\frac { 1 } { N + 1 } \left[\left(k_{2}+i k_{1}\right) a_{1}^{\dagger}\right.\right. \\
& \left.\left.+\left(k_{4}-i k_{3}\right) a_{2}^{\dagger}\right] \frac{1}{N+1} e^{i k \cdot \hat{x}}\right]
\end{aligned}
$$

where we have used the relation (3.4). Similarly we can evaluate the other terms as

$$
\begin{aligned}
\operatorname{Tr}\left[E e^{i A}\right]= & \operatorname{Tr}\left[\frac{N}{N+1} k \cdot \hat{x} \frac{1}{N+1} e^{i k \cdot \hat{x}}\right] \\
& -\frac{1}{\sqrt{2}} \operatorname{Tr}\left[\frac { 1 } { N + 1 } \left[\left(k_{2}+i k_{1}\right) a_{1}^{\dagger}\right.\right. \\
& \left.\left.+\left(k_{4}-i k_{3}\right) a_{2}^{\dagger}\right] \frac{1}{N+1} e^{i k \cdot \hat{x}}\right],
\end{aligned}
$$

$$
\operatorname{Tr}\left[F e^{i A}\right]=\operatorname{Tr}\left[G e^{i A}\right]=-\operatorname{Tr}\left[\frac{1}{N+1} k \cdot \hat{x} e^{i k \cdot \hat{x}}\right]
$$

Combining these together, we find that the third term is actually zero:

$$
\operatorname{Tr}\left[(D+E+F+G) e^{i A}\right]=0 .
$$

Combining Eqs. (5.17), (5.19), and (5.23), the D0-brane density is given by

$$
J(k)=2 \delta^{4}(k)+1-\frac{1}{4} \rho^{2} k^{2} \int_{0}^{1} d \tau \exp \left(-\frac{k^{2}}{4} \tau^{2}\right)+\mathcal{O}\left(\rho^{4}\right) .
$$

Written in the $x$ representation by the Fourier transformation, the D0-brane density is

$$
\begin{aligned}
J(x)= & \frac{2}{\theta^{2}}+\delta^{4}(x)+\frac{\partial^{2}}{\partial x^{i} \partial x^{i}} \frac{4 \pi^{2} \rho^{2}}{\theta^{2}} \int_{0}^{1} d \tau \frac{1}{r^{4}} \exp \left(-\frac{|x|^{2}}{\tau^{2} \theta}\right) \\
& +\mathcal{O}\left(\frac{\rho^{4}}{\theta^{2}}\right) .
\end{aligned}
$$

Here we have restored $\theta$ using the dimensional analysis and the convention that the parameter $\rho$ has the dimension of length.

Let us interpret this result. The first term in Eq. (5.25) is for the uniformly bounded D0 brane in the D4 brane, and the delta function in the second term represents the D0 brane of 
zero size. Turning on $\rho$ deforms this delta-function singularity. When $x \ll \sqrt{\theta}$, we can evaluate the $\tau$ integral in the third term as

$$
\begin{gathered}
\frac{\partial^{2}}{\partial x^{i} \partial x^{i}} \frac{4 \pi^{2} \rho^{2}}{\theta^{2}} \int_{0}^{1} d \tau \frac{1}{\tau^{4}} \exp \left(-\frac{|x|^{2}}{\tau^{2} \theta}\right) \\
=\rho^{2} \frac{\pi^{5 / 2}}{\sqrt{\theta}} \frac{\partial^{2}}{\partial x^{i} \partial x^{i}} \frac{1}{|x|^{3}}+\mathcal{O}(1) .
\end{gathered}
$$

Therefore, for $|x| \ll \sqrt{\theta}$, the D0 brane density of the noncommutative instanton is

$$
\begin{aligned}
J(x)-\frac{2}{\theta^{2}} & =\delta^{4}(x)+\rho^{2} \frac{\pi^{5 / 2}}{\sqrt{\theta}} \frac{\partial^{2}}{\partial x^{i} \partial x^{i}} \frac{1}{|x|^{3}}+\cdots \\
& =\frac{-1}{2 \pi^{2}} \frac{\partial^{2}}{\partial x^{i} \partial x^{i}}\left(\frac{1}{|x|^{2}}-\rho^{2} \frac{2 \pi^{9 / 2}}{\sqrt{\theta}|x|^{3}}\right)+\cdots, \\
(|x| \ll \sqrt{\theta}) . &
\end{aligned}
$$

Thus the delta-function singularity in the $\rho=0$ solution is modified, suggesting that the singularity is resolved for finite $\rho$. One can imagine, for example, that Eq. (5.27) represents the first two terms in the $\rho$ expansion of the smooth function

$$
\frac{\partial^{2}}{\partial x^{i} \partial x^{i}} \frac{1}{\left(|x|+\rho^{2} / \sqrt{\theta}\right)^{2}},
$$

where we neglected numerical coefficients. We will see in the next section that, for large $\rho$, the D0 brane density $J(x)$ indeed has a smooth profile.

On the other hand, for $|x| \gg \sqrt{\theta}$, the $\tau$ integral in Eq. (5.25) can also be evaluated and the D0-brane density is given by

$$
\begin{gathered}
J(x)-\frac{2}{\theta^{2}}=\frac{2 \pi^{2} \rho^{2}}{\theta} \frac{\partial^{2}}{\partial x^{i} \partial x^{i}}\left[\frac{1}{|x|^{2}} \exp \left(-\frac{|x|^{2}}{\theta}\right)\right]+\cdots, \\
(|x| \gg \sqrt{\theta}) .
\end{gathered}
$$

Thus the asymptotic behavior of the D0 brane charge distribution is Gaussian with the width $\sim \sqrt{\theta}$.

The $U(1)$ part of the field strength, i.e., the D2 brane density, can be evaluated in a similar fashion. Using the expansion

$$
\left[\Psi^{\dagger} a_{1} \Psi, \Psi^{\dagger} a_{1}^{\dagger} \Psi\right]=U_{0}^{\dagger} U_{0}+\frac{\rho^{2}}{2}\left(\begin{array}{cc}
\frac{1}{(N+1)(N+2)} & 0 \\
0 & |0,0\rangle\langle 0,0|-\frac{1}{N_{\neq 0}(N+2)}
\end{array}\right)+\mathcal{O}\left(\rho^{4}\right),
$$

we have

$$
\left.\operatorname{Tr}\left[\left[\Psi^{\dagger} a_{1} \Psi, \Psi^{\dagger} a_{1}^{\dagger} \Psi\right] e^{i k \cdot X}\right]=\operatorname{Tr}\left[U_{0}^{\dagger} U_{0} e^{i k \cdot X}\right]+\rho^{2} \operatorname{Tr}\left[\begin{array}{cc}
\frac{1}{(N+1)(N+2)} & 0 \\
0 & |0,0\rangle\langle 0,0|-\frac{1}{N_{\neq 0}(N+1)}
\end{array}\right] e^{i A}\right]+\mathcal{O}\left(\rho^{4}\right)
$$

The first term in the right-hand side is evaluated in the same fashion, and the result is

$$
\begin{aligned}
\operatorname{Tr}\left[U_{0}^{\dagger}\right. & \left.U_{0} e^{i k \cdot X}\right] \\
= & \delta^{4}(k)+\frac{\rho^{2}}{2}\left[-\int_{0}^{1} d \tau \operatorname{Tr}\left[C e^{i \tau A} B\right]\right. \\
& \left.+\int_{0}^{1} d \tau^{\prime} \tau^{\prime} \int_{0}^{1} d \tau \operatorname{Tr}\left[C e^{i \tau \tau^{\prime} A} B\right]\right]+\mathcal{O}\left(\rho^{4}\right)
\end{aligned}
$$

$$
\begin{aligned}
= & \delta^{4}(k)+\frac{\rho^{2}}{2}\left[-\int_{0}^{1} d \tau \frac{|k|^{2}}{2} e^{-\tau^{2}|k|^{2} / 4}\right. \\
& \left.+\int_{0}^{1} d \tau^{\prime} \tau^{\prime} \int_{0}^{1} d \tau \frac{|k|^{2}}{2} e^{-\tau^{2} \tau^{\prime 2}|k|^{2} / 4}\right]+\mathcal{O}\left(\rho^{4}\right) .
\end{aligned}
$$

The second term in Eq. (5.32) turns out to be simple,

$$
\frac{\rho^{2}}{2}\left(1-e^{-|k|^{2} / 4}\right) \text {. }
$$


Summing up all the contributions and noting that the second integral in Eq. (5.32) is arranged to cancel with the error function coming from the first integral, we found that the result vanishes:

$$
\operatorname{Tr}\left[\left(\Psi^{\dagger} a_{1} \Psi, \Psi^{\dagger} a_{1}^{\dagger} \Psi\right) e^{i k \cdot X}\right]=\delta^{4}(k)+0+\mathcal{O}\left(\rho^{4}\right)
$$

Therefore, the Seiberg-Witten transform of the $U(1)$ part of the field strength vanishes

$$
\operatorname{tr} F_{34}(x)=0+\mathcal{O}\left(\rho^{4}\right) .
$$

Similarly one can show that all other components vanish to this order,

$$
\operatorname{tr} F_{i j}(x)=0+\mathcal{O}\left(\rho^{4}\right) .
$$

In fact one can show that, if $\operatorname{tr} F_{i j}$ is smooth and decays sufficiently fast at infinity, it vanishes identically,

$$
\operatorname{tr} F_{i j}=0 \text {. }
$$

To see this, we note that the anti-self-dual equation,

$$
\left[X^{i}, X^{j}\right]=-\frac{1}{2} \epsilon_{i j k l}\left[X^{k}, X^{l}\right]
$$

implies, via the Seiberg-Witten map, that $\operatorname{tr} F_{i j}$ is also antiself-dual. Since $\operatorname{tr} F_{i j}$ obeys the Bianchi identity as shown in Ref. [46], we can write $\operatorname{tr} F_{i j}=\partial_{\left[i, a_{j}\right]}$ for some $U(1)$ gauge field $a_{i}$. It is well-known that there is no nontrivial solution to the anti-self-dual equation in the $U(1)$ gauge theory. Thus it should vanish identically for any $\rho$, assuming it is smooth and vanishes sufficiently fast for large $x$. One can also argue that the BPS instanton solution considered here should not carry any local D2 brane charges. The computation at large $\rho$, in the next section, also shows that $\operatorname{tr} F_{i j}$ vanishes.

\section{B. Large $\rho$ expansion}

Before going into a detailed calculation of the large $\rho$ expansion, let us take a look at the limit $\rho=\infty$. There we have

$$
X^{\mu}=\hat{x}^{\mu} I_{2 \times 2} .
$$

Note that the nonzero contribution is coming from the second term of the solution (5.4), not from the first term, which dominates in the opposite limit $\rho=0$. It is clear that the Seiberg-Witten map gives zero gauge field and vanishing D0 brane density. This is consistent with the expectation that, in the large $\rho$ limit, the instanton spreads over and the structure of the soliton disappears.

Now let us evaluate the subleading terms in the $1 / \rho$ expansion,

$$
\Psi^{\dagger} k \cdot \hat{x} \Psi=k \cdot \hat{x}+\frac{2}{\rho^{2}} P+\frac{4}{\rho^{4}} Q+\frac{8}{\rho^{6}} R+\mathcal{O}\left(1 / \rho^{8}\right),
$$

where

$$
P \equiv \frac{1}{2} k \cdot \hat{x} \otimes\left(\begin{array}{cc}
1 & 0 \\
0 & -1
\end{array}\right)+\left(\begin{array}{cc}
0 & p_{2} a_{1}-p_{1} a_{2} \\
\left(p_{2} a_{1}-p_{1} a_{2}\right)^{\dagger} & 0
\end{array}\right),
$$

$$
Q \equiv \frac{-3}{8} k \cdot \hat{x} \otimes \mathbb{1}_{2 \times 2}-\frac{1}{2} P(N+1)-\frac{1}{2}(N+1) P,
$$

$\operatorname{tr} R=\frac{3}{4}(N k \cdot \hat{x}+k \cdot \hat{x} N+2 k \cdot \hat{x})$.

In Eq. (5.41), we used the complex combination of the momentum $k$ defined as

$$
p_{1}=\frac{1}{\sqrt{2}}\left(k_{2}+i k_{1}\right), \quad p_{2}=\frac{1}{\sqrt{2}}\left(k_{4}-i k_{3}\right) .
$$

We did not write down the explicit form of $R$ since only its $U(2)$ trace, $\operatorname{tr} R$, is going to be necessary in the following. To evaluate $Q$ and $\operatorname{tr} R$, we have used the relation

$$
[N,[N, k \cdot \hat{x}]]=k \cdot \hat{x} .
$$

Let us compute the D0-brane density

$$
J(k) \equiv \operatorname{Tr}\left[\exp \left(i \Psi^{\dagger} k \cdot \hat{x} \Psi\right)\right] .
$$

It turns out that the $\mathcal{O}\left(\rho^{-2}\right)$ term vanishes since tr $P=0$. Thus we have to start with the $\mathcal{O}\left(\rho^{-4}\right)$ terms.

Using the cyclic property of the trace $\mathbf{T r}$, we find

$$
\begin{aligned}
\left.\frac{1}{4} J(k)\right|_{\operatorname{order}\left(1 / \rho^{4}\right)} & =\operatorname{Tr}\left[i Q e^{i k \cdot \hat{x}}\right]+\operatorname{Tr} \sum_{n=0}^{\infty} \sum_{l, m \geqslant 0}\left[(i k \cdot \hat{x})^{l} i P(i k \cdot \hat{x})^{m} i P(i k \cdot \hat{x})^{n-2-l-m}\right] \\
& =\operatorname{Tr}\left[i Q e^{i k \cdot \hat{x}}\right]+\operatorname{Tr}\left[(i P)^{2} e^{i k \cdot \hat{x}}\right],
\end{aligned}
$$


where we used the fact that $P$ and $k \cdot \hat{x}$ commute. To evaluate the traces, we employ the following formulas proven in Appendix A:

$$
a_{1} e^{i k \cdot \hat{x}}=-i\left(\frac{\partial}{\partial \bar{p}_{1}}-\frac{1}{2} p_{1}\right) e^{i k \cdot \hat{x}}, \text { etc, }
$$

where $p_{1}, p_{2}$ are the complex combination of the momentum (5.44). The result is

$$
\begin{aligned}
\left.\frac{1}{4} J(k)\right|_{\text {order } 1 / \rho^{4}} & 8 \delta^{4}(k)+\left(\left|p_{1}\right|^{2} \frac{\partial}{\partial p_{2}} \frac{\partial}{\partial \bar{p}_{2}}+\left|p_{2}\right|^{2} \frac{\partial}{\partial p_{1}} \frac{\partial}{\partial \bar{p}_{1}}\right. \\
& \left.-\bar{p}_{1} p_{2} \frac{\partial}{\partial p_{2}} \frac{\partial}{\partial \bar{p}_{1}}-\bar{p}_{2} p_{1} \frac{\partial}{\partial p_{1}} \frac{\partial}{\partial \bar{p}_{2}}\right) \delta^{4}(k) .
\end{aligned}
$$

This is further simplified by

$$
p_{1} \frac{\partial}{\partial p_{1}} \delta^{4}(k)=-\delta^{4}(k),
$$

and finally we obtain

$$
\left.J(k)\right|_{\text {order } 1 / \rho^{4}}=\frac{24}{\rho^{4}} \delta^{4}(k) .
$$

Therefore, in terms of the commutative $x$ coordinates, the $\mathcal{O}\left(\rho^{-4}\right)$ term in the D0-brane density is

$$
\left.J(x)\right|_{\text {order } 1 / \rho^{4}}=\frac{24}{\rho^{4}} .
$$

Remarkably, this agrees with the $1 / \rho$ expansion of the BPST instanton in the commutative gauge theory:

$$
F_{\mu \nu}=\frac{4 \rho^{2}}{\left(|x|^{2}+\rho^{2}\right)^{2}} \Sigma_{\mu \nu}
$$

where $\Sigma_{\mu \nu} \equiv \eta^{i \mu \nu} \sigma_{i}$ with the Pauli matrix $\sigma_{i}(i=1,2,3)$ and the 't Hooft symbol $\eta$. Substituting this into the D0-brane density

$$
\frac{1}{8} \operatorname{tr} \epsilon^{i j k l}\left(F_{i j}-\theta_{i j}^{-1}\right)\left(F_{k l}-\theta_{k l}^{-1}\right)
$$

and expanding it in powers of $1 / \rho$, we find

$$
\begin{aligned}
& -\frac{1}{8} \operatorname{tr} \epsilon^{i j k l}\left(F_{i j}-\theta_{i j}^{-1} 1\right)\left(F_{k l}-\theta_{k l}^{-1} 1\right) \\
& =\frac{2}{\theta^{2}}+\frac{1}{8} \operatorname{tr} \epsilon^{i j k l} F_{i j} F_{k l} \\
& =\frac{2}{\theta^{2}}+\frac{24}{\rho^{4}}-\frac{96}{\rho^{6}}|x|^{2}+\mathcal{O}\left(\frac{1}{\rho^{8}}\right) .
\end{aligned}
$$

The $\mathcal{O}\left(\rho^{-4}\right)$ term exactly agrees with the above calculation (5.52).
The fact that the noncommutative instanton becomes the commutative one in the limit $\theta \rightarrow 0$ does not by itself guarantee this agreement. For example, there could have been a correction of the form $e^{-x^{2} / \theta}$ multiplying $\rho^{-4}$, which vanishes in the commutative limit. Such a correction is absent since the structure of the expansion given by Eqs. (5.41) (5.43) suggests that the coefficients of the $1 / \rho$ expansion are polynomials in $x$. By a simple dimensional analysis, one can show that, under this condition, no $\theta$ dependent term is allowed in the $0\left(\rho^{-4}\right)$ order. Therefore the agreement of the number 24 gives a nice consistency check of our computation.

We have gone further and carried out the $\mathcal{O}\left(\rho^{-6}\right)$ computation of the D0 brane density. The detail is given in Appen$\operatorname{dix} \mathrm{B}$. The result is even more surprising:

$$
\left.J(x)\right|_{\text {order } 1 / \rho^{6}}=-\frac{96}{\rho^{6}}|x|^{2} .
$$

This term perfectly agrees with the corresponding term in Eq. (5.55). Thus, even to this order, there are no corrections to the D0 brane distribution due to the noncommutativity. We should point out that, to this order, there could have been a term of the form $\theta / \rho^{6}$, but the coefficient in front of it turned out to be zero.

We have also computed the $U(1)$ part of the field strength, i.e., the D2-brane density. The leading term is of the order $\mathcal{O}\left(\rho^{-2}\right)$, but it turned out to be zero, in agreement with expectation that the BPS instanton does not carry any D2brane charge.

\section{CONCLUSION}

In this paper, we have evaluated the Seiberg-Witten map for various solitons and instantons in noncommutative gauge theory. When the gauge theory is defined by the low energy limit of string theory, the Seiberg-Witten map describes how these solutions couple to the Ramond-Ramond potentials of closed string theory [46-48]. Therefore, by studying the Seiberg-Witten map, we can read off various information about Ramond-Ramond charge distributions of these solutions.

We find that the Ramond-Ramond charge distributions of solutions, constructed using projection operators, have deltafunction supports. They include solutions in two-dimensional Yang-Mills theory (3.1), pure D0 brane in various dimensions (3.7), intersecting D2 branes (4.1), (4.7), (4.8), (4.16), (4.17), and D1 branes intersecting with D3 brane (4.20).

On the other hand, instantons in higher dimensions allow deformation away from the projection operator construction and therefore their Seiberg-Witten transforms can have finite sizes. We studied in detail the case of the $U(2)$ anti-self-dual instanton given by Eqs. (5.4) and (5.5). The solution has the deformation parameter $\rho$. In the limit $\rho \rightarrow 0$, the solution reduces to the one for the pure D0 brane (3.7). Turning on a small amount of $\rho$, the D0-brane density is deformed as in Eq. (5.25). We see that the D0-brane charge is now distributed over the region of size $\sim \sqrt{\theta}$. In addition, the delta- 
function singularity of the D0-brane charge distribution is modified as

$$
\delta(x)=\frac{-1}{2 \pi^{2}} \frac{\partial^{2}}{\partial x^{i} \partial x^{i}} \frac{1}{|x|^{2}} \rightarrow \frac{-1}{2 \pi^{2}} \frac{\partial^{2}}{\partial x^{i} \partial x^{i}}\left(\frac{1}{|x|^{2}}-\rho^{2} \frac{2 \pi^{9 / 2}}{\sqrt{\theta}|x|^{3}}\right) .
$$

For large $\rho$, we can evaluate the Seiberg-Witten map of the instanton in the $1 / \rho$ expansion. We find that the D0-brane density of the noncommutative instanton agrees surprisingly well with that of the commutative instanton. The agreement in the leading terms, Eqs. (5.52) and (5.55), is expected and gives a nice consistency check of our computation. The agreement of the subleading term, Eqs. (5.56) and (5.55), is surprising and we do not have an explanation for this phenomenon.

We also find that the $U(1)$ part of the Seiberg-Witten map vanishes for both small $\rho$ and large $\rho$. Since there is no nontrivial anti-self-dual solution in the $U(1)$ gauge theory in commutative space, we expect that $\operatorname{tr} F_{i j}$ vanishes for any $\rho$. It is consistent with the expectation that the BPS instanton should not carry any local D2-brane charges.

In Refs. [61-65] the Seiberg-Witten transform of noncommutative monopoles are studied with fixed $\alpha^{\prime}$ and small $\theta$. This is in contrast to our case where we use the exact Seiberg-Witten map of [46-48] in the Seiberg-Witten limit $\left(\alpha^{\prime} \rightarrow 0\right.$ ) and with finite $\theta$. It will be interesting to extend this analysis to include the case studied in Refs. [61-65].

In this paper, we have evaluated the Seiberg-Witten map for the $U(1)$ part of the field strength. It is desirable to find an explicit expression for the non-Abelian part of the Seiberg-Witten map since it would carry more information on these solutions. Progress in this direction has been made in Refs. [66], [67]. (For our purpose, we need an inverse of the map studied in these papers.)

\section{ACKNOWLEDGMENTS}

We thank Yuji Okawa for useful discussions and for comments on the earlier version of this paper. H.O. thanks the Institute for Theoretical Physics, Santa Barbara, for the hospitality. K.H. was supported in part by Japan Society for the Promotion of Science under the Postdoctoral Research Program (\#02482). H.O. was supported in part by the Department of Energy Grant DE-FG03-92ER40701 and the Caltech Discovery Fund. In addition, this research was supported in part by the National Science Foundation under Grant No. PHY99-07949.

\section{APPENDIX A: USEFUL FORMULAS}

In this appendix we derive the formula (5.48) and other useful formulas used in the evaluation of the large $\rho$ expansion in Sec. V B. We find it useful to introduce the complex combinations of the momentum $k$ as

$$
p_{1}=\frac{1}{\sqrt{2}}\left(k_{2}+i k_{1}\right), \quad p_{2}=\frac{1}{\sqrt{2}}\left(k_{4}-i k_{3}\right)
$$

so that the following relation holds:

$$
k \cdot \hat{x}=p_{1} a_{1}^{\dagger}+\bar{p} a_{1}+p_{2} a_{2}^{\dagger}+\bar{p}_{2} a_{2} .
$$

To show Eq. (5.48) is easy, by acting a derivative on $e^{i k \cdot \hat{x}}$ as

$$
\begin{aligned}
\frac{\partial}{\partial \bar{p}_{1}} e^{i k \cdot \hat{x}} & =\sum_{n} \frac{(i)^{n}}{n !} \sum_{m=0}^{n-1}(k \cdot \hat{x})^{m} a_{1}(k \cdot \hat{x})^{n-1-m} \\
& =\sum_{n} \frac{(i)^{n}}{n !}\left(n a_{1}(k \cdot \hat{x})^{n-1}+\sum_{m=0}^{n-1}\left(-p_{1}\right)(k \cdot \hat{x})^{n-2}\right) \\
& =i a_{1} e^{i k \cdot \hat{x}}+\frac{1}{2} p_{1} e^{i k \cdot \hat{x}} .
\end{aligned}
$$

This verifies Eq. (5.48).

In the following, we shall derive a useful formula that is necessary in evaluating the $1 / \rho^{6}$ contribution in the D0-brane density in Appendix B. For simplicity we consider two dimensional noncommutative space and evaluate

$$
\operatorname{Tr}\left[n e^{i k \cdot \hat{x}}\right]
$$

Taking the derivative twice, we easily obtain

$$
\operatorname{Tr}\left[a^{\dagger} a e^{i k \cdot \hat{x}}\right]=-i\left(\frac{\partial}{\partial \bar{p}}-\frac{1}{2} p\right)\left[-i\left(\frac{\partial}{\partial p}+\frac{1}{2} \bar{p}\right) \delta^{2}(k)\right] .
$$

Here note the order of the differentiation. Taking care of the formula

$$
x \partial_{x} \delta(x)=-\delta(x)
$$

we obtain

$$
\operatorname{Tr}\left[a^{\dagger} a e^{i k \cdot \hat{x}}\right]=\left(-\frac{1}{2}-\frac{\partial}{\partial p} \frac{\partial}{\partial \bar{p}}\right) \delta^{2}(k)
$$

Therefore, for $N \equiv a_{1}^{\dagger} a_{1}+a_{2}^{\dagger} a_{2}$, we obtain

$$
\operatorname{Tr}\left[N e^{i k \cdot \hat{x}}\right]=\left(-1-\frac{\partial}{\partial p_{1}} \frac{\partial}{\partial \bar{p}_{1}}-\frac{\partial}{\partial p_{2}} \frac{\partial}{\partial \bar{p}_{2}}\right) \delta^{4}(k)
$$

\section{APPENDIX B: EVALUATION OF ORDER $\rho^{-6}$ TERMS IN THE $U(2)$ INSTANTON}

In this appendix, we derive the subleading result (5.56).

The contribution of this order $\mathcal{O}\left(1 / \rho^{6}\right)$ in the D0-brane current density $\exp \left[i \Psi^{\dagger} k \cdot \hat{x} \Psi\right]$ is 


$$
\begin{aligned}
& 8 \sum_{n} \frac{i^{n}}{n !}\left(\sum_{m_{1}, m_{2}, m_{3} \geqslant 0} \operatorname{Tr}(k \cdot \hat{x})^{m_{1}} P(k \cdot \hat{x})^{m_{2}} P(k \cdot \hat{x})^{m_{3}} P(k \cdot \hat{x})^{n-3-m_{1}-m_{2}-m_{3}}\right. \\
& \quad+\sum_{m_{1}, m_{2} \geqslant 0} \operatorname{Tr}(k \cdot \hat{x})^{m_{1}} P(k \cdot \hat{x})^{m_{2}} Q(k \cdot \hat{x})^{n-2-m_{1}-m_{2}}+\sum_{m_{1}, m_{2} \geqslant 0} \operatorname{Tr}(k \cdot \hat{x})^{m_{1}} Q(k \cdot \hat{x})^{m_{2}} P(k \cdot \hat{x})^{n-2-m_{1}-m_{2}} \\
& \left.\quad+\sum_{m \geqslant 0} \operatorname{Tr}(k \cdot)^{m} R(k \cdot \hat{x})^{n-1-m}\right)
\end{aligned}
$$

Using the cyclic property of the trace under that these summation over $n$ can be expressed in terms of the compact operator $e^{i k \cdot \hat{x}}$, and we can rewrite this as ${ }^{7}$

$$
8 \operatorname{Tr}\left[(i P)^{3} e^{i k \cdot \hat{x}}+(i Q)(i P) e^{i k \cdot \hat{x}}+i R e^{i k \cdot \hat{x}}\right] .
$$

Let us evaluate each term in the trace, respectively.

The first term turns out to be vanishing. This is because

$$
\operatorname{tr}\left[P^{3}\right]=\frac{1}{4}(k \cdot \hat{x}) k^{2}
$$

and thus

$$
\operatorname{Tr}\left[(i P)^{3} e^{i k \cdot \hat{x}}\right]=-\frac{1}{4} k^{2} \operatorname{Tr}\left[i k \cdot \hat{x} e^{i k \cdot \hat{x}}\right]=k^{2} \delta^{4}(k)=0 .
$$

The second term in Eq. (B3) is calculated in the following. First, taking the $U(2)$ trace, we have

$$
\begin{aligned}
\operatorname{Tr}\left[Q P e^{i k \cdot \hat{x}}\right]= & -\operatorname{Tr}\left[( N + 1 ) \left(\frac{1}{2}(k \cdot \hat{x})^{2}+\frac{1}{2} k^{2}+2\left(\bar{p}_{2} a_{1}^{\dagger}\right.\right.\right. \\
& \left.\left.\left.-\bar{p}_{1} a_{2}^{\dagger}\right)\left(p_{2} a_{1}-p_{1} a_{2}\right)\right) e^{i k \cdot \hat{x}}\right]
\end{aligned}
$$

Using the formula (A 8), the first term of this expression is evaluated as

$$
\begin{aligned}
& \operatorname{Tr}\left[(N+1) \frac{1}{2}(k \cdot \hat{x})^{2} e^{i k \cdot \hat{x}}\right] \\
& \quad=-\left.\frac{1}{2}\left(\frac{\partial}{\partial \tau}\right)^{2} \operatorname{Tr}\left[(N+1) e^{i \tau k \cdot \hat{x}}\right]\right|_{\tau=1}
\end{aligned}
$$

${ }^{7}$ For example, the last term in Eq. (B1) is rearranged without using the cyclicity as

$$
\int_{0}^{1} d \tau \operatorname{Tr}\left[e^{i \tau k \cdot \hat{x}} \operatorname{Re}^{i(1+-\tau) k \cdot \hat{x}}\right] .
$$

However, concerning the first term in Eq. (B1), it is not necessary to use the cyclic property because $P$ is commutative with $k \cdot \hat{x}$.

$$
\begin{aligned}
= & -\frac{1}{2}\left(\frac{\partial}{\partial \tau}\right)^{2}\left[\left(-\frac{\partial}{\partial\left(\tau p_{1}\right)} \frac{\partial}{\partial\left(\tau \bar{p}_{1}\right)}\right.\right. \\
& \left.\left.-\frac{\partial}{\partial\left(\tau p_{2}\right)} \frac{\partial}{\partial\left(\tau \bar{p}_{2}\right)}\right) \delta^{4}(\tau k)\right]\left.\right|_{\tau=1} \\
= & 21\left(\frac{\partial}{\partial p_{1}} \frac{\partial}{\partial \bar{p}_{1}}+\frac{\partial}{\partial p_{2}} \frac{\partial}{\partial \bar{p}_{2}}\right) \delta^{4}(k) .
\end{aligned}
$$

We calculate the second term in the similar way and obtain

$$
\operatorname{Tr}\left[\frac{1}{2}(N+1) k^{2} e^{i k \cdot \hat{x}}\right]=-2 \delta^{4}(k)
$$

The third term is slightly complicated; however, using the formulas (5.48) and (A8) the straightforward calculation shows

$$
\begin{aligned}
& \operatorname{Tr}\left\{(N+1)\left[\left(\bar{p}_{2} a_{1}^{\dagger}-\bar{p}_{1} a_{2}^{\dagger}\right)\left(p_{2} a_{1}-p_{1} a_{2}\right)\right] e^{i k \cdot \hat{x}\}}\right. \\
&=-\bar{p}_{1} p_{1}\left(\frac{\partial}{\partial \bar{p}_{2}}-\frac{1}{2} p_{2}\right)\left(\frac{\partial}{\partial p_{2}}+\frac{1}{2} \bar{p}_{2}\right) \\
&-\bar{p}_{2} p_{2}\left(\frac{\partial}{\partial \bar{p}_{1}}-\frac{1}{2} p_{1}\right)\left(\frac{\partial}{\partial p_{1}}+\frac{1}{2} \bar{p}_{1}\right) \\
&+\bar{p}_{2} p_{1}\left(\frac{\partial}{\partial \bar{p}_{2}}-\frac{1}{2} p_{2}\right)\left(\frac{\partial}{\partial p_{1}}+\frac{1}{2} \bar{p}_{1}\right) \\
&\left.+\bar{p}_{1} p_{2}\left(\frac{\partial}{\partial \bar{p}_{1}}-\frac{1}{2} p_{1}\right)\left(\frac{\partial}{\partial p_{2}}+\frac{1}{2} \bar{p}_{2}\right)\right] \\
& \times\left(-\frac{\partial}{\partial p_{1}} \frac{\partial}{\partial \bar{p}_{1}}-\frac{\partial}{\partial p_{2}} \frac{\partial}{\partial \bar{p}_{2}}\right) \delta^{4}(k) \\
&=\left(1-3 \frac{\partial}{\partial p_{1}} \frac{\partial}{\partial \bar{p}_{1}}-3 \frac{\partial}{\partial p_{2}} \frac{\partial}{\partial \bar{p}_{2}}\right) \delta^{4}(k) .
\end{aligned}
$$

Therefore, summarizing them, we have

$$
\operatorname{Tr}(i Q)(i P) e^{i k \cdot \hat{x}}=15\left(\frac{\partial}{\partial p_{1}} \frac{\partial}{\partial \bar{p}_{1}}+\frac{\partial}{\partial p_{2}} \frac{\partial}{\partial \bar{p}_{2}}\right) \delta^{4}(k) .
$$

The third term in Eq. (B3) is rather easily evaluated by using Eq. (A8), and the result is 


$$
\operatorname{Tr}(i R) e^{i k \cdot \hat{x}}=9\left(\frac{\partial}{\partial p_{1}} \frac{\partial}{\partial \bar{p}_{1}}+\frac{\partial}{\partial p_{2}} \frac{\partial}{\partial \bar{p}_{2}}\right) \delta^{4}(k)
$$

Summing up all the contributions (B5), (B10), and (B11), we obtain the order $1 / \rho^{6}$ result as

$$
\frac{192}{\rho^{6}}\left(\frac{\partial}{\partial p_{1}} \frac{\partial}{\partial \bar{p}_{1}}+\frac{\partial}{\partial p_{2}} \frac{\partial}{\partial \bar{p}_{2}}\right) \delta^{4}(k) .
$$

Restoring the $\theta$ dependence and noting the relations

$$
\frac{\partial}{\partial p_{1}} \frac{\partial}{\partial \bar{p}_{1}}=\frac{1}{2}\left[\frac{\partial^{2}}{\partial k_{1} \partial k_{1}}+\frac{\partial^{2}}{\partial k_{2} \partial k_{2}}\right],
$$

we obtain

$$
\left.J(k)\right|_{\operatorname{order}\left(\theta^{3} / \rho^{6}\right)}=\frac{96}{\rho^{6}} \frac{\partial^{2}}{\partial k_{i} \partial k_{i}} \delta^{4}(k) .
$$

Performing the Fourier transformation, we obtain the result (5.56).
[1] A. Connes, M. R. Douglas, and A. Schwarz, J. High Energy Phys. 02, 003 (1998).

[2] N. Nekrasov and A. Schwarz, Commun. Math. Phys. 198, 689 (1998).

[3] K. Furuuchi, Prog. Theor. Phys. 103, 1043 (2000)

[4] K.-Y. Kim, B.-H. Lee, and H. S. Yang, "Comments on Instantons on Noncommutative $\mathrm{R}^{4}$," hep-th/0003093.

[5] K. Furuuchi, Commun. Math. Phys. 217, 579 (2001).

[6] D. J. Gross and N. A. Nekrasov, J. High Energy Phys. 07, 034 (2000)

[7] A. P. Polychronakos, Phys. Lett. B 495, 407 (2000).

[8] D. J. Gross and N. Nekrasov, J. High Energy Phys. 10, 021 (2000).

[9] C. Sochichiu, J. High Energy Phys. 08, 026 (2000).

[10] D. Bak, Phys. Rev. B 495, 251 (2000).

[11] M. Aganagic, R. Gopakumar, S. Minwalla, and A. Strominger, J. High Energy Phys. 04, 001 (2001).

[12] K. Furuuchi, "Topological Charge of U(1) Instantons," hep-th/0010006.

[13] N. A. Nekrasov, "Noncommutative instantons revisited," hep-th/0010017.

[14] J. A. Harvey, P. Kraus, and F. Larsen, J. High Energy Phys. 12, 024 (2000).

[15] D. J. Gross and N. Nekrasov, J. High Energy Phys. 03, 044 (2001).

[16] K. Furuuchi, J. High Energy Phys. 03, 033 (2001).

[17] M. Hamanaka and S. Terashima, J. High Energy Phys. 03, 034 (2001).

[18] K. Hashimoto, J. High Energy Phys. 12, 023 (2000).

[19] M. Mihailescu, I. Y. Park, and T. A. Tran, Phys. Rev. D 64, 046006 (2001).

[20] D. Bak, K. Lee, and J.-H. Park, Phys. Rev. D 63, 125010 (2001).

[21] B. Durhuus, T. Jonsson, and R. Nest, Phys. Lett. B 500, 320 (2001).

[22] G. S. Lozano, E. F. Moreno, and F. A. Schaposnik, Phys. Lett. B 504, 117 (2001).

[23] S. P. de Alwis and A. T. Flournoy, Phys. Rev. D 63, 106001 (2001).

[24] D. Bak, K. Lee, and J. H. Park, Phys. Lett. B 501, 305 (2001).

[25] A. Hashimoto and N. Itzhaki, Phys. Rev. D 63, 126004 (2001).

[26] S. Corley and S. Ramgoolam, J. High Energy Phys. 03, 037 (2001).

[27] C. Sochichiu, "Exercising in K-theory: Brane Condensation without Tachyon," hep-th/0012262.
[28] G. S. Lozano, E. F. Moreno, and F. A. Schaposnik, J. High Energy Phys. 02, 036 (2001).

[29] A. Bergman, O. J. Ganor, and J. L. Karczmarek, Phys. Rev. D 64, 065001 (2001).

[30] L.-S. Tseng, "Noncommutative Solitons and Intersecting D-Branes," hep-th/0101125.

[31] K. Hashimoto and K. Krasnov, Phys. Rev. D 64, 046007 (2001).

[32] A. Khare and M. B. Paranjape, J. High Energy Phys. 04, 002 (2001).

[33] D. Bak, K. Lee and J.-H. Park, Phys. Rev. Lett. 87, 030402 (2001).

[34] D. Bak and K. Lee, Phys. Lett. B 509, 168 (2001).

[35] O. Lechtenfeld, A. D. Popov, and B. Spendig, J. High Energy Phys. 06, 011 (2001).

[36] M. G. Jackson, “The Stability of Noncommutative Scalar Solitons," hep-th/0103217.

[37] R. Gopakumar, M. Headrick, and M. Spradlin, "On Noncommutative Multi-solitons," hep-th/0103256.

[38] L. Hadasz, U. Lindstrom, M. Rocek, and R. von Unge, J. High Energy Phys. 06, 040 (2001).

[39] C. Sochichiu, "Some Notes Concerning the Dynamics of Noncommutative Solitons in the M(atrix) Theory as well as in the Noncommutative Yang-Mills," hep-th/0104076.

[40] M. Rangamani, "Reverse Engineering ADHM Construction from Non-Commutative Instantons," hep-th/0104095.

[41] C. Acatrinei and C. Sochichiu "A note on the decay of noncommutative solitons," hep-th/0104263.

[42] T. Araki and K. Ito, "Scattering of Noncommutative $(n, 1)$ Solitons," hep-th/0105012.

[43] A. Fujii, Y. Imaizumi, and N. Ohta, "Supersymmetry, Spectrum, and Fate of D0-Dp Systems with B-field," hep-th/0105079.

[44] D. H. Correa, G. S. Lozano, E. F. Moreno, and F. A. Schaposnik, "Comments on the U(2) Noncommutative Instanton," hep-th/0105085.

[45] B. Chen and F.-L. Lin, "Tachyon Condensation of D2/D4 Brane System in Noncommutative Gauge Theory," hep-th/0105154.

[46] Y. Okawa and H. Ooguri, Phys. Rev. D 64, 046009 (2001).

[47] S. Mukhi and N. V. Suryanarayana, J. High Energy Phys. 05, 023 (2001).

[48] H. Liu and J. Michelson, "Ramond-Ramond Couplings of Noncommutative D-branes," hep-th/0104139.

[49] J. A. Harvey, "Topology of the Gauge Group in Noncommu- 
tative Gauge Theory," hep-th/0105242.

[50] D. J. Gross, A. Hashimoto, and N. Itzhaki, "Observables of Non-Commutative Gauge Theories,” hep-th/0008075.

[51] N. Seiberg and E. Witten, J. High Energy Phys. 09, 032 (1999).

[52] K. Okuyama, J. High Energy Phys. 03, 016 (2000).

[53] B. Jurco and P. Schupp, Eur. Phys. J. C 14, 367 (2000).

[54] B. Jurco, P. Schupp, and J. Wess, Nucl. Phys. B584, 784 (2000).

[55] B. Jurco, P. Schupp, and J. Wess, Nucl. Phys. B604, 148 (2001).

[56] M. Kontsevich, "Deformation quantization of Poisson manifolds, I,” q-alg/9709040.

[57] A. S. Cattaneo and G. Felder, Commun. Math. Phys. 212, 591 (2000)
[58] H. Liu, “*-Trek II: $*_{\mathrm{n}}$ operations, open Wilson lines and the Seiberg-Witten map," hep-th/0011125.

[59] N. Ishibashi, S. Iso, H. Kawai, and Y. Kitazawa, Nucl. Phys. B573, 573 (2000).

[60] S. R. Das and S. Rey, Nucl. Phys. B590, 453 (2000).

[61] D. Mateos, Nucl. Phys. B577, 139 (2000).

[62] K. Hashimoto and T. Hirayama, Nucl. Phys. B587, 207 (2000).

[63] S. Moriyama, Phys. Lett. B 485, 278 (2000).

[64] S. Moriyama, J. High Energy Phys. 08, 014 (2000).

[65] S. Goto and H. Hata, Phys. Rev. D 62, 085022 (2000).

[66] B. Jurco, L. Moller, S. Schraml, P. Schupp, and J. Wess, "Construction of non-Abelian Gauge Theories on Noncommutative Spaces," hep-th/0104153.

[67] D. Brace, B. L. Cerchiai, A. F. Pasqua, U. Varadarajan, and B. Zumino, J. High Energy Phys. 06, 047 (2001). 